\title{
NUMERICAL SIMULATION OF STOCHASTIC DIFFERENTIAL EQUATIONS USING IMPLICIT MILSTEIN METHOD
}

\author{
Ratna Herdiana \\ Department of Mathematics, Diponegoro University \\ Email: ratnaherdiana@lecturer.undip.ac.id
}

\begin{abstract}
Stiff stochastic differential equations arise in many applications including in the area of biology. In this paper, we present numerical solution of stochastic differential equations representing the Malthus population model and SIS epidemic model, using the improved implicit Milstein method of order one proposed in [6]. The open source programming language SCILAB is used to perform the numerical simulations. Results show that the method is more accurate and stable compared to the implicit Euler method.
\end{abstract}

Keywords: Stochastic differential equations, implicit Milstein method, SCILAB.

\section{INTRODUCTION}

In early development of mathematical models, it is common to describe the behavior of physical phenomena using deterministic differential equations that ignores the effects of intrinsic noise. Such equations represent idealized situation and less realistic. In the last 3 decades, we find many mathematical models in various fields [1] [2], such as, biology, physics, finance, economy and environmental sciences modelling that incorporate the intrinsic noise in the model so as to achieve more natural representation.

The stochastic ordinary differential equations have been used in these circumstances. Stochastic differential equations (SDE) include a random term which describes the intrinsic noise, or randomness, within the dynamical system. In the SDE, a Wiener process is a mathematical tool, used to model the motion of individual particles. The Wiener process is widely found in mathematical models involving various noisy systems. It is associated with the British botanist Robert Brown, thus also called the Brownian motion.

A scalar standard Brownian motion, or standard Wiener process, over time interval [0, T] is a random variable $W(t)$ that depends continuously on $t \in[0, T]$ and satisfies the following three conditions.

1. $W(0)=0$ (with probability 1$)$.

2. For $0 \leq s<t \leq T$ the random variable given by the increment $W(t)-W(s)$ is normally distributed with mean zero and variance $t-s$; equivalently, $W(t)-W(s) \sim \sqrt{t-s} N(0,1)$ where $N(0,1)$ denotes a normally distributed random variable with zero mean and unit variance.

3. For $0 \leq s<t<u<v \leq T$ the increments $W(t)-W(s)$ and $W(v)-W(u)$ are independent. 
The stochastic differential equations are interpreted in the framework of Itô Calculus [1].

A stochastic process $X(\mathrm{t})$ is an Itô stochastic ordinary differential equation (SDE) written as

$$
\begin{aligned}
& d X=f(X(t)) d t+\sum_{i=1}^{d} g_{i}(X(t)) d W_{i}(t), \quad X(t) \in \mathrm{R}^{m}, 0 \leq t \leq T \\
& X(0)=X_{0}
\end{aligned}
$$

where $T$ is a positive constant, $f: \mathrm{R}^{m} \rightarrow \mathrm{R}^{m} \quad$ is the drift coefficient, and $g_{i}: \mathrm{R}^{m} \rightarrow \mathrm{R}^{m}, \quad i=1, \ldots, d$, are the diffusion coefficient. The increment $d W_{i}(t)$ of the Wiener process $W_{i}(t), i=1, \ldots, d$ are independent and normally distributed $N(0, \Delta t)$. The initial condition $X_{0}$ is independent of the Wiener process satisfying $\mathrm{E}\left[X_{0}\right]^{2}<\infty$.

Since in the majority cases analytical or explicit solutions of SDE are not available, numerical methods become increasingly important in many applications to simulate and approximate the mathematical models. There are two types of numerical methods, the explicit methods and the implicit methods. The explicit method [11] is best used for non-stiff problems. A widely used explicit method to solve strong approximation of (1) is the Euler-Maruyama (EM) method of order 0ne-half [6]. However, EM method will not give strong solution for SDEs with super linearly growing drift coefficient. Explicit methods when used to solve stiff problems has poor stability properties and need very small step size, hence computational costs increased.

One of the early implicit methods for stiff stochastic differential equations was introduced in [5] known as the balanced implicit method with better stability properties than the explicit methods. A combination of semi-implicit and implicit Milstein method called the composite Milstein method was introduced in [10] for strong solution of (1) adapting the same technique to construct the composite Euler [9]. The composite Milstein scheme is an alternative method to solve stiff SDE since the convergence rate and stability of the composite Milstein investigated in [10] showed to be better than the composite Euler.

The implicit methods have better stability properties and well adapted for stiff problems [11]. A number of numerical schemes for solving non-linear SDE with better stability have been developed. In [13] an improved Milstein (IM) method was constructed by adding an error correction term to the Milstein method. The method was proved to be more efficient and has very large stability region, moreover it avoids solving nonlinear algebra equations encountered in other implicit methods. Furthermore, in [7] two extension of semi-implicit Milstein method was introduced, drift-implicit and double-implicit Milstein method for non-linear SDE. Both methods converge to exact solution with strong order one and preserves stability.

The intention in this paper is to demonstrate numerical solution of the SDE that models biological phenomena using the implicit numerical scheme, namely the strong order 0.5 implicit Euler (IE) method and order 1 modified implicit Milstein (IM) method introduced in [13]. Detail theoretical back ground can be found in the literature [11]. In the next section, we briefly present these two numerical scheme.

The classic Malthus population growth model with environmental randomness will be considered as example to visualize the difference between true solution and solution when IE method and IM method are used. This is given in section three. We also implement the 
improved IM methods to the general SIS epidemic model which incorporate demographic randomness in the model. We concentrate on strong solution of SDEs, such solution is considered for the following reasons: when investigating the dependence on the initial conditions or the values of parameters that define the SDE.

The available open source software SCILAB ver. 6.0.2 is used for all computations and simulations and support the results with graphs and tables. Program list of the sce-files are given at the end.

\section{NUMERICAL METHODS}

For SDE (1), we define a uniform finite time interval in $[0, T]$ with step size $\Delta t=T / n, n \in \mathbb{N}^{+}$by $0=t_{0}<t_{1}<\cdots<t_{n}=T$. The numerical methods give approximate value of the sample path step-by-step at discrete time $t_{i}, i=1,2, \ldots, n$. In some applications the solution is required for one realization $(d=1)$ of the given Wiener path- called path wise or strong solution, the SDE is thus written

$$
d X(t)=f(X(t)) d t+g(X(t)) d W(t), \quad X(0)=X_{0}, 0 \leq t \leq T
$$

where $f$ and $g$ are scalar functions and the initial condition $X_{0}$ is a random variable. The solution $X(t)$ is a random variable for each $t$.

In a system for which different components evolve on different time scales, the implicit methods allow to capture the dynamics of the system on the slow time scale without resolving the transient effects on the fast time scale.

Here we present the Implicit Euler method [8] of strong order 0.5 and the Implicit Milstein method of strong order 1 which have been modified to provide better accuracy [13].

\section{The implicit Euler method.}

The implicit numerical scheme is implicit in both the drift coefficients and the diffusion coefficients. One step of an implicit Euler for the SDE (2) has convergence order $1 / 2$ and is written in the form

$$
X_{i}=X_{i-1}+f\left(X_{i}\right) \Delta t+g\left(X_{i}\right) \Delta W_{i-1}
$$

where $X_{i}=X\left(t_{i}\right)$ and $\Delta W_{i} \sim N(0, \Delta t)$ for $i=0,1, \ldots, N$. In-depth study on the stability and properties of Implicit Euler method is found in [8]. It was found that the method does not converge to the Itô SDE but rather to the corresponding right point SDE. However, it is a reasonable method as it can behave like Itô solution when the noise intensity is large or very small. The method is asymptotically stable for linear SDE problem.

[8] It seems that implicit methods may be used in special cases such as linear equations with a strongly attracting drift and a very weak diffusion coefficient. For SDE which are stiff in both deterministic and stochastic term the IE method is not recommended.

The modified implicit Milstein method.

The modified implicit Milstein method proposed in [13] is briefly represented here. Let $X_{1} \approx X\left(t_{1}\right)$ the approximate solution of (2) at $t_{1}$, and $\hat{X}_{1}$ the corresponding approximate 
solution when explicit Milstein method is applied to (2). The difference $\bar{q}_{1}=X_{1}-\hat{X}_{1}$ is then used and approximated as correction for the semi-implicit Milstein method [11]. The construction in [13] resulted $\bar{q}_{1}=\left(1-\Delta t f^{\prime}\left(\widehat{X}_{1}\right)\right)^{-1} \Delta t\left(f\left(\widehat{X}_{1}\right)-f\left(X_{0}\right)\right)$.

The modified implicit Milstein method introduced is thus consist of two steps, define as

$$
\left\{\begin{array}{l}
\hat{X}_{j}=X_{j-1}+\Delta t f\left(X_{j-1}\right)+g\left(X_{j-1}\right) \Delta W_{j-1}+\frac{1}{2} g^{\prime}\left(X_{j-1}\right) g\left(X_{j-1}\right)\left(\Delta W_{j-1}^{2}-\Delta t\right) \\
X_{j}=\hat{X}_{j}+\left(1-\Delta t f^{\prime}\left(\hat{X}_{j}\right)\right)^{-1} \Delta t\left(f\left(\hat{X}_{j}\right)-f\left(X_{j-1}\right)\right)
\end{array}\right.
$$

It was shown the method has strong convergence of order one in mean-square sense, additional advantage is the scheme does not require solving nonlinear algebraic problem common associated with implicit methods.

The IM method is found to be suitable for stiff mean-square problems with moderate stochastic noise intensity or additive noise where the drift coefficient is dominant in the dynamics of the model.

\section{NUMERICAL SIMULATION}

There are two classes of SDE models in biology, first the models which consider on environmental randomness that may affect the parameters. The second class considers on natural demographic randomness to exist even when the growing rate is constant and the environment disturbances are disregarded. The second class model are usually non-linear, hence therefore for this reason the use of numerical simulation is important in order to have some insight about the behavior of the model.

Here we consider two SDE model, one from each class and provide numerical simulation using the implicit Euler and implicit Milstein scheme discussed in previous section.

\subsection{The Malthus population growth model}

Consider a growing population of cells or animals at a constant rate $r$, in a bounded habitat with no food shortage. The growing rate per capita, $r$, is affected by small random environmental factor, e.g. temperature, humidity light or diseases. So the linear SDE of the Malthus population model is

$$
\begin{aligned}
& d X(t)=r X(t)+\mu X(t) d W(t) \quad t \in[0, T] \\
& X(0)=X_{0}
\end{aligned}
$$

where $\mu$ is the intensity of the random effects on the growing rate.

The equation has the following exact solution,

$$
X(t)=X_{0} \exp \left(\left(r-\frac{1}{2} \mu^{2}\right) t+\mu W(t)\right)
$$

which is also known as the geometric Brownian motion. The parameter values are set to $r=1$ and two values of noise intensity $\mu=1$ and 0.4 . The initial value $X_{0}=1$. We apply the IM method as well as the IE method on interval $[0,1]$ and calculate the error from the exact solution at the end point $T=1$, to examine which method has better approximation. 
To apply the numerical methods, first construct the Brownian motion. For computational purposes the Brownian motion path is discretized, where $W(t)$ is specified at discrete $t$ values. Set the time increment $\delta t=T / n$ for some positive integer $n$ and let $W_{j}$ denote $W\left(t_{j}\right)$ with $t_{j}=j \delta t . W_{0}=0$ with probability 1 , and $W_{j}=W_{j-1}+d W_{j}, j=1,2, \ldots, n$, where each $d W_{j}$ is an independent random variable of the form $\sqrt{\delta t} N(0,1)$.

In SCILAB, in order to get or set the seed of the random number generator, the function rand is used with simple syntax

$$
\text { rand ('seed', } s \text { ) }
$$

this sets the seed to the value $s$ (by default $\mathrm{s}=0$ at first call). Here we set rand('seed', 10), this allows to produce the same set of random numbers in each run. We provide random number generator of size 1-by- $n$ with distribution $N(0,1)$ : rand $(1, n$, 'normal') which then scaled by $\sqrt{\delta t}$ to create increment $d W$. Then the Wiener path $W$ is obtained by the cumulative sum of $d W$ to form the exact solution (5). The same Wiener path is then used when applying the IE and IM method.

The implicit Euler method for solving (4) takes the form:

$$
X_{j}=X_{j-1}+r X_{j} \Delta t+\mu X_{j} \Delta W_{j-1}
$$

here, for computation convenience the time step $\Delta t=k \delta t$, with $k \geq 1$, is an integer multiple of the increment $\delta t$.In the implementation we take $k=4$, the corresponding Wiener increment with time step $\Delta t=k \delta t$ is computed as [2]

$$
\begin{aligned}
\Delta W_{j} & =W\left(\tau_{j}\right)-W\left(\tau_{j-1}\right) \\
& =W(j k \delta t)-W((j-1) k \delta t)=\sum_{i=(j-1) k+1}^{j k} d W_{i}
\end{aligned}
$$

and written as value of winc.

The implementation of IM method applied to (4) is given by

$$
\left\{\begin{array}{l}
\hat{X}_{j}=X_{j-1}+r X_{j-1} \Delta t+\mu X_{j-1} \Delta W_{j-1}+0.5 \mu^{2} X_{j-1}\left(\Delta W_{j-1}^{2}-\Delta t\right) \\
X_{j}=\hat{X}_{j}+1 /(1-\Delta t r)\left(\hat{X}_{j}-X_{j-1}\right) r
\end{array}\right.
$$

The Scilab file IE_IM.sce performs one simulation of the Malthus model (4) with parameters $r=2, \mu=1$ and constant initial value $X_{0}=1$ using IE and IM method, also the exact solution is computed. The path from three solutions are plotted in Figure 1(a). Figure 1(b) is obtained when the intensity noise is reduced to $\mu=0.4$ and $\delta t=2^{-8}$. The single Brownian motion is generated for each different $\delta t$. The difference between the exact solution and each solution from IE method and IM method at the endpoint $t=T=1$ are calculated for different $\delta t$. These values are summarized in Table. 1 and 2. In the case of large noise $\mu=1$ (Table 1), as expected the IE method is not suitable, as $\delta t$ decreases the discrepancy with the exact solution at $T$ increased, while the IM method show decreased error and better accuracy compared to result in Table 2. 
Table 1. Difference between exact solution and the numerical methods: IE and IM, at the end point $\mathrm{T}=1$. Parameter values: $r=2, \mu=1$

\begin{tabular}{cccc}
\hline & $\delta t=2^{-6}$ & $\delta t=2^{-8}$ & $\delta t=2^{-10}$ \\
\hline IE method & 1.1218 & 1.8228 & 1.8691 \\
\hline IM method & 0.1233 & 0.0399 & 0.0104 \\
\hline
\end{tabular}

Table 2. Difference between exact solution and the numerical methods: IE and IM, at the end point $\mathrm{T}=1$. Parameter values: $r=2, \mu=0.4$

\begin{tabular}{cccc}
\hline & $\delta t=2^{-6}$ & $\delta t=2^{-8}$ & $\delta t=2^{-10}$ \\
\hline IE method & 0.7777 & 0.7173 & 0.6616 \\
\hline IM method & 0.5194 & 0.1338 & 0.0327 \\
\hline
\end{tabular}

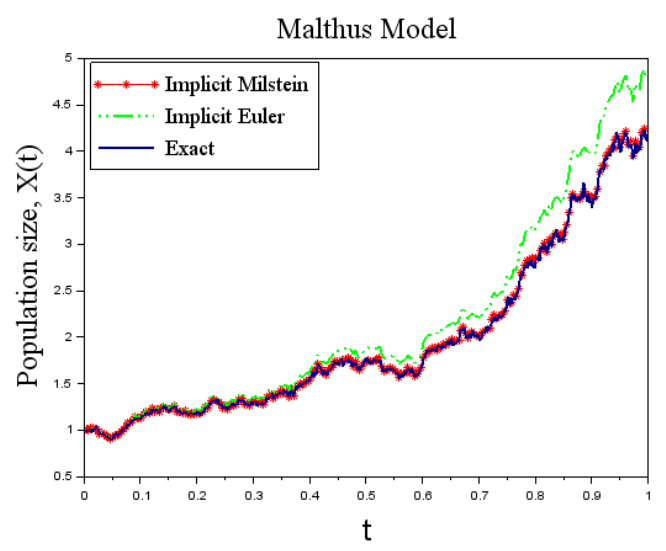

Figure 1. (a) $r=2, \mu=1, \delta \mathrm{t}=2^{\wedge}(-10)$

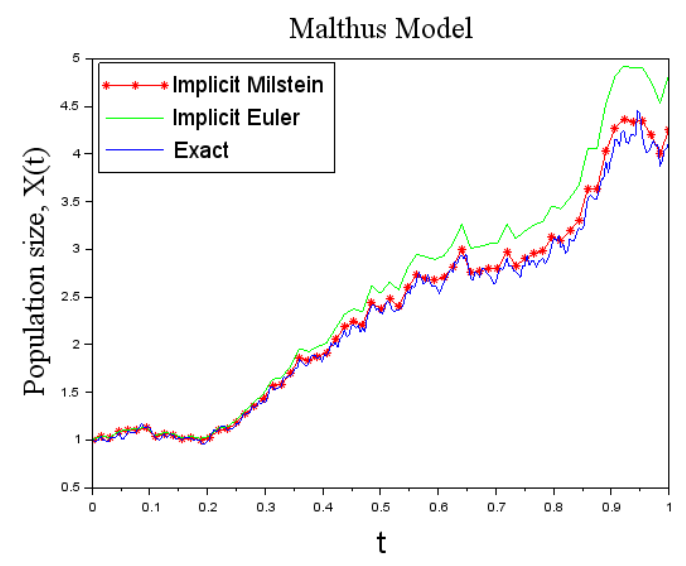

(b) $r=2, \mu=0.4, \delta \mathrm{t}=2^{\wedge}(-8)$

Figure 1 shows plotted graph from the three solutions for the case (a) $\mu=1, \delta t=2^{-10}$ and (b) $\mu=0.4, \delta t=2^{-8}$. It is seen that IM provide far more accurate result compared to the IE scheme. The IM solution matches closely to the exact solution as the time step $\Delta t$ decreases.

\subsection{The SIS epidemic model}

An epidemic model depicts the dynamic associated to the transmission of a disease within a population. Here we consider the basic epidemic model, the SIS model which describes the number of susceptible $(S)$, infected $(I)$ individuals of a constant population of size $N$. The following assumptions are made: a susceptible individual, after a successful contact with an infectious individual, becomes infected and infectious, but does not develop immunity to the disease. This means, after recovery, an infected individuals return to the susceptible group. All 
individuals have the same probability of contracting the disease at a rate proportional to the number of meetings between infected and susceptible, $\beta S I$. There is no disease related deaths.

The deterministic SIS epidemic model is defined:

$$
\begin{aligned}
& \frac{d S}{d t}=-\frac{\beta}{N} S I+(b+\gamma) I \\
& \frac{d I}{d t}=\frac{\beta}{N} S I-(b+\gamma) I,
\end{aligned}
$$

where the contact rate: $\beta>0$; recovery rate: $\gamma>0$; and the birth rate: $b \geq 0$. The birth rate and death rate is assumed to be equal, hence the population size remain constant, $d N / d t=0$.

The formulation of a SDE for the SIS epidemic model may be derived from the continuous time Markov chain SIS epidemic model [3]. Since $S(t)=N-I(t)$, only one independent variable is in the SIS model, that is $I(t)$ the number of infected. The assumptions in the SIS epidemic model are in terms of the variation $\Delta I=I(t+\Delta t)-I(t)$.

Let $U(I)=U(I(t))=\beta I(t)(N-I(t)) / N ; \quad$ and $\quad V(I)=V(I(t))=(b+\gamma) I(t)$.

The transition probability of three possible state is assumed

$$
P\{\Delta I=j \mid I(t)=i\}=\left\{\begin{array}{lr}
U(i) \Delta t+o(\Delta t), & j=i+1 \\
V(i) \Delta t+o(\Delta t), & j=i-1 \\
1-[U(i)+V(i)] \Delta t+o(\Delta t), & j=i \\
o(\Delta t), & j \neq i+1, i, i-1
\end{array}\right.
$$

It is assume that the changes in the number of infected individuals is maximum one. The state $j=i+1$ represent new infection occur or a birth, $j=i-1$ means a death or recovery occurred, while no changes is stated $j=i$. Since the population size is kept constant, a birth of a susceptible is followed by a death, so no need of the case $j=i$ in the deterministic or stochastic formulation.

The expectation and the variance of $\Delta I$ are computed as follows [3],

$$
\begin{aligned}
E(\Delta I) & =U(I) \Delta t-V(I) \Delta t+o(\Delta t)=[U(I)-V(I)] \Delta t+o(\Delta t) \\
& =\mu(I) \Delta t+o(\Delta t) \\
\operatorname{Var}(\Delta I) & =E(\Delta I)^{2}-[E(\Delta I)]^{2}=U(I) \Delta t+V(I) \Delta t+o(\Delta t) \\
& =[U(I)+V(I)] \Delta t+o(\Delta t)=\sigma^{2}(I) \Delta t+o(\Delta t)
\end{aligned}
$$

Since the random variable $\Delta I$ is approximately normally distributed for small $\Delta t$, $\Delta I(t) \sim N\left(\mu(I) \Delta t, \sigma^{2}(I) \Delta t\right)$, then

where $\omega \sim N(0,1)$.

$$
\begin{aligned}
I(t+\Delta t) & =I(t)+\Delta I(t) \\
& \approx I(t)+\mu(I) \Delta t+\sigma(I) \sqrt{\Delta t} \omega,
\end{aligned}
$$

Therefore the Ito SDE of the SIS epidemic is written as: 


$$
d I=\mu(I) d t+\sigma(I) d W
$$

where $W$ is the Wiener process, $W(t+\Delta t)-W(t)=\Delta W \sim N(0, \Delta t)$. The coefficients of the stochastic SIS epidemic model are $\mu(I)=U(I)-V(I)$ and $\sigma(I)=\sqrt{U(I)+V(I)}$. In the case of disease free equilibrium, $I(t)=0$, it can be seen that $d I / d t=0$ which is an absorbing state for the Itô SDE.

To apply a numerical method to (6) over [0,25], firstly discretize the interval. Let $\Delta t=k \delta t$ for positive integer $k=1,4$, and $\tau_{j}=j \Delta t$. We denote $I_{j}$ as the numerical approximation to $I\left(\tau_{j}\right)$, the number of infected at time $\tau_{j}$.

The modified implicit Milstein applied to (6) then takes the form

$$
\left\{\begin{array}{l}
\hat{I}_{j}=I_{j-1}+U\left(I_{j-1}\right) \Delta t+V\left(I_{j-1}\right) \Delta W_{j-1}+\frac{1}{2} V^{\prime}\left(I_{j-1}\right) V\left(I_{j-1}\right)\left(\Delta W_{j-1}^{2}-\Delta t\right) \\
I_{j}=\hat{I}_{j}+\left(1-\Delta t U^{\prime}\left(\hat{I}_{j}\right)\right)^{-1} \Delta t\left(U\left(\hat{I}_{j}\right)-U\left(I_{j-1}\right)\right.
\end{array}\right.
$$

The parameter values are set as $b=0.25, \gamma=0.25, \beta=1$, and population size $N=100$. The initial condition is $I(0)=2$. The basic reproduction number is $R_{0}=2$, so that the deterministic solution approaches the endemic equilibrium $\bar{I}=50$. We simulate the IM scheme (7) for two different time step, $2^{\wedge}(-8)$, over $M=100$ sample paths, then take the average. The function mean is used to compute the average. Figure 2(a) shows the plotted averaged path and the corresponding mean deterministic solution, where mean- 1 and mean- 2 correspond to average path when $k=1$ and $k=2$ respectively. In the case $k=1, \delta t=2^{-8}$, from the 100 paths we plotted five sample paths shown in figure 3 . The difference at the end point $T$ between the average solution by IM method and the deterministic solution is computed as ER. In figure 2 (a), ER $=1.6323$ for mean- 1 and $E R=1.2438$ for mean- 2 .

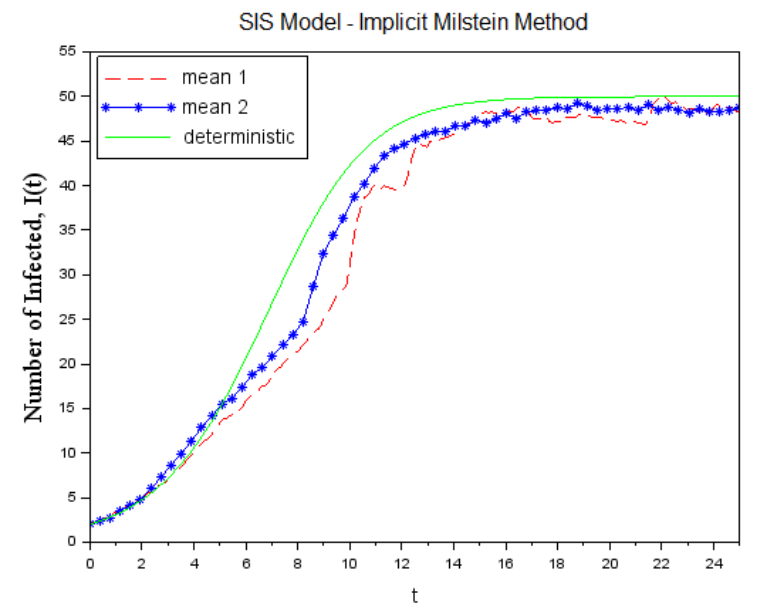

Figure 2. (a) $\beta=1, M=100, \delta t=2^{\wedge}(-8)$

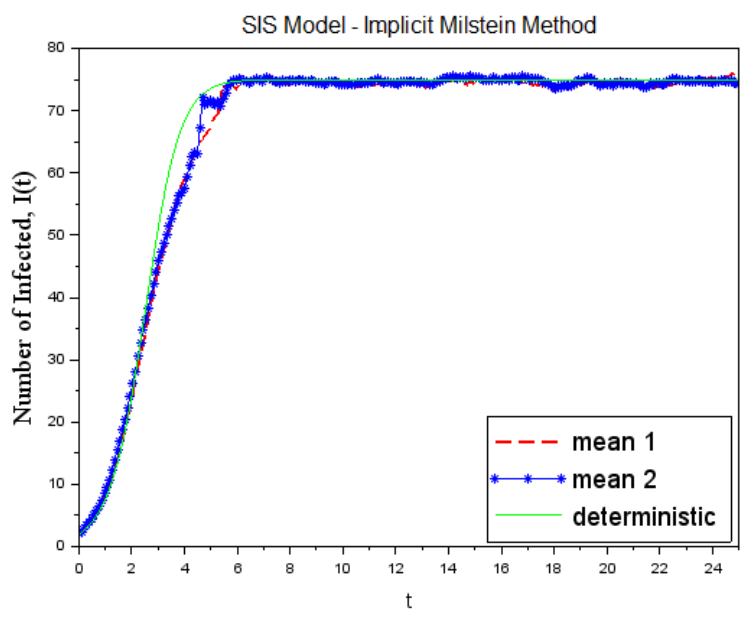

(b) $\beta=2, M=100, \delta t=2^{\wedge}(-10)$ 
The simulation is repeated for the case when stiffness is increased $\beta=2$, with increment $\delta t=2^{-10}$. Then $R_{0}=4$ and the endemic equilibrium reached $\bar{I}=75$ is seen in figure 2 (b). The ER is computed for mean- 1 and mean-2, $\mathrm{ER}=0.9130$ and $\mathrm{ER}=0.7745$ respectively.

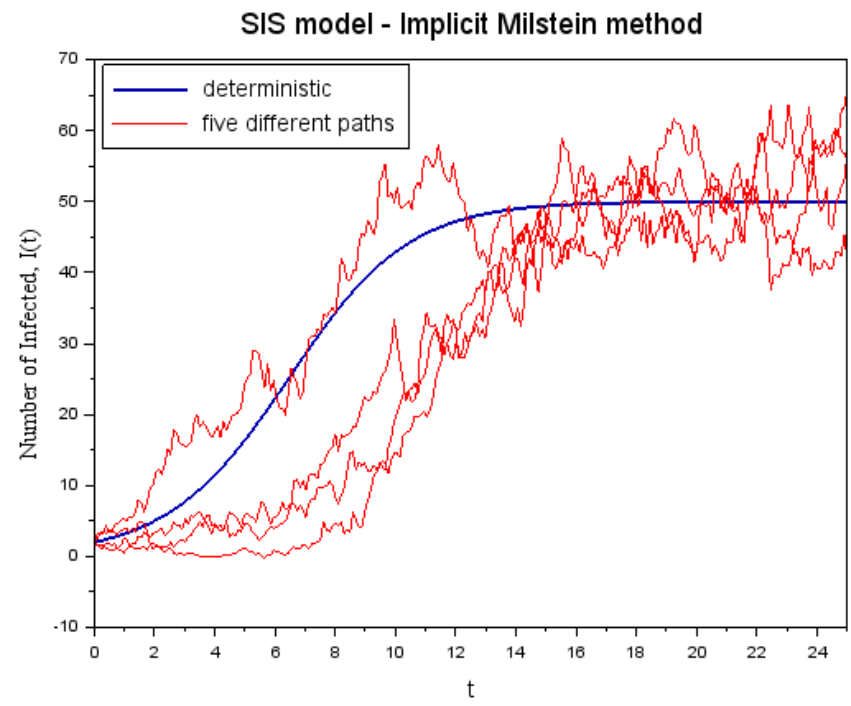

Figure 3. Five sample paths

The averaged sample path follows closely the dynamics of the corresponding deterministic solution with the same time-step and the same value of parameters. From the plotted graphs, we can visualize the fact suggested that the average (mean) function converges to the solution of the deterministic model.

The above simulations are obtained from the Scilab program file IMSIS.sce provided in Listing 2.

\section{CONCLUSION}

We have demonstrated application of modified implicit method [13] to simulate approximated solutions of linear and non-linear SDE model. The convincing results show that the method is more accurate and stable compared to the IE method. In the case of solving nonlinear SDE the averaged solution by the IM method, over 100 paths, closely follows the corresponding deterministic solution and almost coincide in the non-stiff part of the solution. For computational efficiency the method may be implemented with adaptive step size. Numerical experiment may also be conducted in applying the method to solve more advance stiff SDE model in biology as well as other areas.

\section{REFERENCES}

[1] B. Øksendal, "Stochastic Differential Equations. An Introduction with Applications", $5^{\text {th }}$ Ed., Springer Verlag Heidelberg, New York, 2000. 
[2] D.J. Higham, "An Algorithmic Introduction to Numerical Simulation of Stochastic Differential Equations", SIAM Review Vol.43, No. 3, pp.525 - 546, 2001.

[3] E. J. Allen, "Stochastic Differential Equations and persistence Time for Two Interacting Populations", Dyn.Cont., Discrete and Impulsive System, 5, pp. 271 - 281, 1999.

[4] G.N. Milstein, "Numerical Integration of Stochastic Differential Equatuions", Springer Science and Business Media, 1994.

[5] G.N. Milstein, E. Platen, H. Schurz: Balanced implicit methods for stiff stochastic systems. SIAM J. Numer. Anal. 35(3), 1010-1019 (1998)

[6] G. Maruyama, "Continuos Markov Process and Stochastic Equations", Rend.Circolo, Math. Parlermo 4(1),1955, 48 - 90.

[7] Jinran Yao, Siqing Gan, "Stability of Drift-Implicit and Double -Implicit Milstein Schemes for Nonlinear Stochastic Diffrential Equations", J. Applied Mathematics and Computation, Vol.339, 2018, pp. 294-301

[8] K. Burrage and T. Tian, "A Note on the Stability Properties of the Euler Methods for Solving Stochastic Differential Equations", New Zealand Journal of Mathematics, Vol. 29, pp.115 - 127, 2000.

[9] K. Burrage, Tian, T, "The composite Euler method for stiff stochastic differential equations". J. Comput. Appl. Math. 131(1-2), 407-426 (2001)

[10 ] M.A. Omar, A. Aboul Hassan, S.I. Rabia, "The CompositeMilstein methods for numerical solution of Ito Stochastic Differential Equations", Journal of Computaional and Applied Mathematics, Vol.235, 2011, pp.2277-2299.

[11] P.E. Kloeden and E. Platen, "The numerical solution of stochastic differential equations", Springer, Berlin, 1999.

[12] X. Mao, "Stochastic Differential Equations and Applications", Howard Publishing Limited, Chichester, 2007.

[13] Z. Yin and S. Gan, "An Improved Milstein Method for Stiff Stochastic Differential Equations", Springer Open Journal, Advances in Difference Equations: 369, 2015.

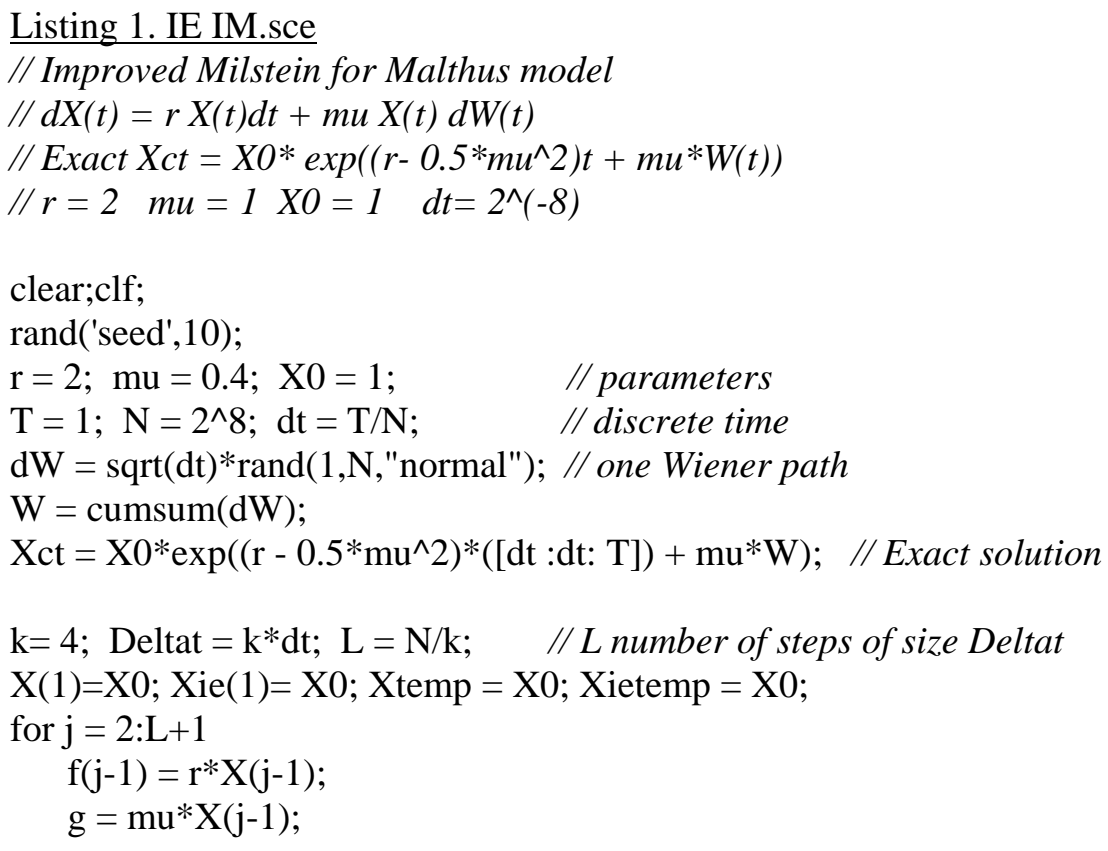


winc $=\operatorname{sum}\left(\mathrm{dW}\left((\mathrm{j}-2) * \mathrm{k}+1:(\mathrm{j}-1)^{*} \mathrm{k}\right)\right)$;

Xtemp $=\mathrm{X}(\mathrm{j}-1)+\mathrm{f}(\mathrm{j}-1) *$ Deltat $+\mathrm{g}^{*}$ winc $+0.5^{*}\left(\right.$ winc $^{\wedge} 2-$ Deltat $) * m u^{*} \mathrm{~g}$;

df $=r ; \quad$ ftemp $=r^{*}$ Xtemp;

$\mathrm{X}(\mathrm{j})=\mathrm{Xtemp}+1 /(1-$ Deltat*df $) *(\mathrm{ftemp}-\mathrm{f}(\mathrm{j}-1)) *$ Deltat;

//Implicit Euler

$\mathrm{C}=1 /\left(1-\mathrm{r}^{*}\right.$ Deltat-mu*winc $)$;

Xietemp = C*Xietemp;

$\operatorname{Xie}(\mathrm{j})=\mathrm{Xietemp}$;

end

plot([0 : Deltat:T], X', 'r-*',[0 : Deltat:T], Xie', 'g-',[0:dt:T], [X0,Xct], 'b-');

legend('Implicit Milstein', 'Implicit Euler','Exact',2)

xlabel('t'); ylabel('X(t)'); title('Malthus Model');

errim $=\operatorname{abs}(X(L+1)-X c t(N))$

errie $=\operatorname{abs}(\mathrm{Xie}(\mathrm{L}+1)-\mathrm{Xct}(\mathrm{N}))$;

Listing 2. IMSIS.sce

// Improved Milstein for SIS model over many Wiener paths

$/ / d I(t)=(\operatorname{beta} * I *(N-I) / N-(b+\operatorname{gama}) I) d t+\operatorname{sqrt}(\operatorname{beta} * I *(N-I) / N+(b+\operatorname{gama}) I) d W(t)$

// beta $=1 \quad$ gama=0.25 $b=0.25 \quad I 0=2 \quad P=100$

clear;clf;

rand('seed',50);

beta $=2 ;$ gama $=0.25 ; \mathrm{I} 0=2 ; \mathrm{b}=0.25 ; \mathrm{P}=100 ; \quad$ // parameters

$\mathrm{T}=25 ; \quad \mathrm{N}=2^{\wedge} 10 ; \mathrm{dt}=\mathrm{T} / \mathrm{N} ; \quad / /$ discretize time

$\mathrm{M}=100 \quad$ // number of Wiener paths

$\mathrm{k}=[1,4]$;

$\mathrm{I}(1)=\mathrm{I} 0 ; \mathrm{dI}(1)=\mathrm{I} 0 ; \mathrm{Itemp}=\mathrm{I} 0$;

for $\mathrm{i}=1: 2$

// different timestep

Deltat $=\mathrm{k}(\mathrm{i}) * \mathrm{dt} ; \mathrm{L}=\mathrm{N} / \mathrm{k}(\mathrm{i}) ; \quad / /$ L number of steps of size Deltat

$\mathrm{AA}=\mathrm{zeros}(\mathrm{M}, \mathrm{L}) ; \quad$ Amean=zeros $(1, \mathrm{~L}) ; \quad \mathrm{dI}=\mathrm{zeros}(\mathrm{L})$; $\mathrm{dI}(1)=\mathrm{I} 0$;

for $\mathrm{m}=1: \mathrm{M} \quad / / M$ different Wiener paths

$\mathrm{dW}=\operatorname{sqrt}(\mathrm{dt}) * \operatorname{rand}(1, \mathrm{~N}, "$ normal");

$\mathrm{I}=\operatorname{zeros}(1, \mathrm{~L}) ; \mathrm{I}(1)=\mathrm{I} 0$;

for $\mathrm{j}=2: \mathrm{L}+1$

winc $=\operatorname{sum}(\mathrm{dW}((\mathrm{j}-2) * \mathrm{k}(\mathrm{i})+1:(\mathrm{j}-1) * \mathrm{k}(\mathrm{i})))$;

$\mathrm{f}(\mathrm{j}-1)=\operatorname{beta}^{*} \mathrm{I}(\mathrm{j}-1) *(\mathrm{P}-\mathrm{I}(\mathrm{j}-1)) / \mathrm{P}-(\mathrm{b}+$ gama $) * \mathrm{I}(\mathrm{j}-1) ; / / d r i f t$

$\mathrm{g}=\operatorname{sqrt}\left(\right.$ beta $^{*} \mathrm{I}(\mathrm{j}-1) *(\mathrm{P}-\mathrm{I}(\mathrm{j}-1)) / \mathrm{P}+(\mathrm{b}+$ gama $\left.) * \mathrm{I}(\mathrm{j}-1)\right) ; / /$ diffusion

$\mathrm{dg}=0.5^{*}($ beta $+\mathrm{b}+$ gama $)-$ beta $* \mathrm{I}(\mathrm{j}-1) / \mathrm{P}$;

Itemp $=\mathrm{I}(\mathrm{j}-1)+\mathrm{f}(\mathrm{j}-1) *$ Deltat $+\mathrm{g} *$ winc $+0.5 *\left(\right.$ winc $^{\wedge} 2-$ Deltat $) * \mathrm{dg}$;

df $=$ beta* $(1-2 *$ Itemp/P) -b-gama;

ftemp $=$ beta $*$ Itemp $*(\mathrm{P}-$ Itemp $) / \mathrm{P}-(\mathrm{b}+$ gama $) *$ Itemp;

$\mathrm{I}(\mathrm{j})=$ Itemp $+1 /(1-$ Deltat $* \mathrm{df}) *(\mathrm{ftemp}-\mathrm{f}(\mathrm{j}-1)) *$ Deltat;

Itemp $=$ Itemp $+1 /(1-$ Deltat $* \mathrm{df}) *($ ftemp $-\mathrm{f}(\mathrm{j}-1)) *$ Deltat;

end

$\mathrm{AA}(\mathrm{m},:)=\mathrm{I}(2: \mathrm{L}+1)$;

end

Amean=mean $(\mathrm{AA}(:, 1: \mathrm{L}), 1)$

//Deterministic solution when $g(I(t))=0$

for $\mathrm{s}=1: \mathrm{L}$

end

$\mathrm{dI}(\mathrm{s}+1)=\mathrm{dI}(\mathrm{s})+($ beta*dI $(\mathrm{s}) *(\mathrm{P}-\mathrm{dI}(\mathrm{s})) / \mathrm{P}-(\mathrm{b}+\mathrm{gama}) * \mathrm{dI}(\mathrm{s})) *$ Deltat; 
$\mathrm{ER}(\mathrm{i})=\operatorname{abs}(\mathrm{Amean}(\mathrm{L})-\mathrm{dI}(\mathrm{L})) ; / /$ difference at end point if $i==1$

else

$\operatorname{plot}([0$ : Deltat:T], [I0,Amean], 'r--','Linewidth',1); end

plot([0 : Deltat:T], [I0,Amean], 'b-*','Linewidth',1);

end

$\operatorname{plot}([0$ : Deltat:T],dI','g-' );

title('SIS model - Implicit Milstein method'); xlabel('t'); ylabel('Number of Infected, I(t)'); legend('mean 1', 'mean 2', 'deterministic',2); 